\title{
Impact of Dual versus Single Antiplatelet Therapy on Major Cardiovascular Outcomes in Patients with Acute Coronary Syndrome in the Arabian Gulf
}

\author{
Ibrahim Al-Zakwani ${ }^{a, b}$ Jawad Al-Lawatic Alawi A. Alsheikh-Alid, e \\ Wael Almahmeed $^{f}$ Wafa Rashed $^{g}$ Arif Al-Mulla ${ }^{h}$ Mohammad Zubaid ${ }^{i}$ \\ a Department of Pharmacology and Clinical Pharmacy, College of Medicine and Health Sciences, Sultan Qaboos \\ University, Muscat, Oman; ${ }^{b}$ Gulf Health Research, Muscat, Oman; ' Directorate General, Primary Health Care, Ministry \\ of Health, Muscat, Oman; ${ }^{d}$ College of Medicine, Mohammed Bin Rashid University of Medicine and Health Sciences,

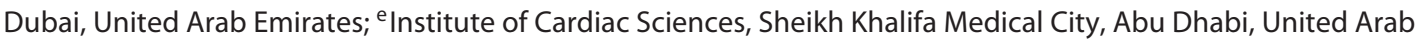 \\ Emirates; ${ }^{\mathrm{f}}$ Heart and Vascular Institute, Cleveland Clinic Abu Dhabi, Abu Dhabi, United Arab Emirates; ${ }^{9}$ Department

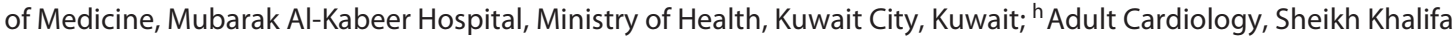 \\ Medical City, Abu Dhabi, United Arab Emirates; 'Department of Medicine, Faculty of Medicine, Kuwait University, \\ Kuwait City, Kuwait
}

\section{Highlights of the Study}

- This study evaluated the impact of dual versus single antiplatelet therapy on major adverse cardiovascular outcomes in Arab citizens with acute coronary syndrome.

- At 12-months follow-up post hospital discharge, the dual antiplatelet regimen was associated with reduced myocardial infarction, mortality, and readmissions for cardiac reasons.

- However, the dual regimen was also associated with increased risk of stroke/transient ischemic attack.

\section{Keywords}

Clopidogrel · Aspirin - Dual antiplatelet therapy · Acute coronary syndrome $\cdot$ Stroke $\cdot$ Myocardial infarction . Mortality · Arabs · Middle East

\footnotetext{
Abstract

Objective: To evaluate the association of dual versus single antiplatelet therapy with major adverse cardiovascular events (MACE) in patients with acute coronary syndrome (ACS) in the Arabian Gulf. Subjects and Methods: Data were analyzed from 3,559 patients with a diagnosis of ACS admitted to 29 hospitals in 4 Arabian Gulf countries (Bahrain, Kuwait, Oman, and United Arab Emirates) from January 2012 to
}

\begin{tabular}{ll}
\hline KARGER & ( ) 2019 The Author(s) \\
& Published by S. Karger AG, Basel Openger \\
karger@karger.com & This is an Open Access article licensed under the Creative Commons \\
www.karger.com/mpp & $\begin{array}{l}\text { Attribution-NonCommercial-4.0 International License (CC BY-NC) } \\
\text { (http://www.karger.com/Services/OpenAccessLicense), applicable to } \\
\text { the online version of the article only. Usage and distribution for com- } \\
\text { mercial purposes requires written permission. }\end{array}$
\end{tabular}

January 2013. Dual antiplatelet therapy (DAPT), consisting of aspirin and clopidogrel, was compared to aspirin alone. MACE included 12-months cumulative stroke/transient ischemic attack (TIA), myocardial infarction (MI), all-cause mortality, and readmissions for cardiac reasons, post discharge. Analyses were performed using multivariable logistic regression. Results: A total of $74 \%(n=2,634)$ of the patients were on DAPT. At 12-month follow-up, patients on DAPT were significantly less likely to experience MACE events (adjusted OR [aOR] 0.73; 95\% Cl: 0.61-0.86; $p<0.001$ ). Lower cardiovascular (CV) event rates were also consistent across the following MACE components; MI (aOR 0.66; 95\% Cl: $0.49-$ $0.88 ; p=0.005$ ), all-cause mortality (aOR 0.69; 95\% Cl: $0.51-$ $0.94 ; p=0.018$ ), and readmissions for cardiac reasons (aOR 
$0.79 ; 95 \% \mathrm{Cl}: 0.66-0.95 ; p=0.011)$. Conversely, DAPT was adversely associated with increased risk of stroke/TIA (aOR $1.68 ; 95 \% \mathrm{Cl}: 1.05-2.69 ; p=0.030)$. Conclusions: DAPT, compared to aspirin therapy alone, was generally associated with better CV outcomes after an ACS event. However, DAPT was adversely associated with increased risk of stroke/TIA in ACS patients in the Arabian Gulf. @ 2019 The Author(s)

Published by S. Karger AG, Basel

\section{Introduction}

Acute coronary syndrome (ACS) is the leading cause of mortality in Asia [1,2]. It is also associated with significant morbidity in the region with over 129 million disability-adjusted life years annually [2]. The use of dual antiplatelet therapy (DAPT), which is usually a combination of aspirin and an oral $\mathrm{P} 2 \mathrm{Y} 12$ inhibitor, has become the management of choice for ACS as recommended by the European Society of Cardiology and the European Association for Cardio-Thoracic Surgery [3] as well as the American College of Cardiology and the American Heart Association Task Force on Clinical Practice Guidelines [4].

The benefits of DAPT in the reduction of cardiovascular (CV) events are well established [5-8]. However, data on the use of DAPT among ACS patients in the Arabian Gulf region are scarce. The Gulf COAST (Gulf Locals with ACS Events) was a prospective, multicountry, multicenter registry of consecutive Gulf citizens hospitalized with the final diagnosis of ACS in 4 Arab Gulf countries [9]. This unique registry of Gulf citizens provides an opportunity to evaluate the management of medication and outcomes among ACS patients in the region. The aim of this study was to evaluate the effect of DAPT on major adverse CV events (MACE) in ACS patients in the Arabian Gulf.

\section{Subjects and Methods}

The methods of the Gulf COAST registry have been published previously [9]; the Gulf COAST registry was a prospective, multicenter, multinational, longitudinal, cohort study of consecutive citizens, from 4 countries in the Arabian Gulf (Bahrain, Kuwait, Oman, and United Arab Emirates) admitted from January 2012 to January 2013 to 29 hospitals with a diagnosis of ACS. The registry enrolled a total of 4,044 patients who were $\geq 18$ years of age with ACS diagnosed according to clinical data standards of the American College of Cardiology (ACC) clinical data standards [10].

In this study, DAPT consisted of aspirin $(81 / 100 \mathrm{mg})$ and clopidogrel $(75 \mathrm{mg})$, while single antiplatelet therapy consisted of aspi- rin (81/100 mg) alone. MACE events included stroke/transient ischemic attack (TIA), myocardial infarction (MI), all-cause mortality, and readmissions for cardiac reasons, captured cumulatively at 12-month after hospital discharge. Data collected included patients' demographics, clinical history, laboratory data, management including medications. Follow-up was performed at 12-month post hospital discharge and was carried out by clinic visits or telephone interviews.

The study was approved by the appropriate institutional and/ or national research Ethics Committee in each of the participating Arabian Gulf countries and has been performed in accordance with the ethical standards as laid down in the 1964 Declaration of Helsinki and its later amendments or comparable ethical standards. Informed consent was obtained from all individual participants included in the study.

\section{Statistical Analysis}

Categorical variables were presented as frequencies and percentages and differences were analyzed using Pearson's $\chi^{2}$ tests. Means and SDs were used to summarize continuous data and analyses were performed using Student $t$ test. The association between DAPT and MACE was evaluated using multiple stepwise logistic regression utilizing the backward-selection method with a significance $p$ value of 0.2 for the removal from the model. The models were adjusted for GRACE risk score for in-hospital mortality (a composite score of age, heart rate, systolic blood pressure [BP], serum creatinine, cardiac arrest at admission, stent thrombus (ST) segment deviation on EKG, abnormal cardiac enzymes, and Killip class), which has been validated in a similar ACS registry in the region [11]. The logistic regression models were also adjusted for gender, body mass index, smoking status, alcohol use, peripheral artery disease, dyslipidemia, diabetes mellitus, prior MI, premature coronary artery disease (CAD), diastolic BP, major bleed (during admission), percutaneous coronary intervention (PCI), discharged diagnosis, and use of cardiac medications at hospital discharge (beta blocker, statin, angiotensin-converting enzyme inhibitor [ACEI], or angiotensin receptor blocker [ARB] and calcium channel blocker $[\mathrm{CCB}])$.

The goodness-of-fit of the logistic models were examined using the Lemeshow and Hosmer [12] goodness-of-fit statistic as well as the $C$-index [13]. An a priori two-tailed level of significance was set at the 0.05 level. Statistical analyses were conducted using STATA version 13.1 (StataCorp., 2013, Stata Statistical Software, College Station, TX, USA).

\section{Results}

Of the 4,044 patients in the Gulf COAST registry, we utilized data of 3,559 patients who were discharged with DAPT $(n=2,634 ; 74 \%)$ or aspirin therapy alone $(n=925$; $26 \%)$ for this analysis. The overall mean age was $60 \pm 12$ years and $67 \%(n=2,373)$ were males. A total of $25 \%$ $(n=876)$ of the patients were current smokers and 3.0\% $(n=105)$ were alcohol consumers. Only $29 \%(n=1,026)$ of the patients had PCI. Out of those that had PCI, $81 \%$ ( $n=833)$ had drug-eluting (DE) stents, $15 \%(n=154)$ had 
Table 1. Demographic, clinical characteristics, and medications at discharge for patients in the Arabian Gulf with ACS stratified by DAPT versus aspirin alone: Findings from Gulf COAST registry

\begin{tabular}{|c|c|c|c|c|}
\hline \multirow[t]{2}{*}{ Characteristic, $n$ (\%) unless specified otherwise } & \multirow[t]{2}{*}{ All $(n=3,559)$} & \multicolumn{2}{|l|}{ Antiplatelet } & \multirow[t]{2}{*}{$p$ value } \\
\hline & & aspirin $(n=925)$ & DAPT $(n=2,634)$ & \\
\hline \multicolumn{5}{|l|}{ Demographic } \\
\hline Age, years, mean $\pm S D$ & $60 \pm 12$ & $60 \pm 13$ & $60 \pm 12$ & 0.121 \\
\hline Gender, male & $2,373(67)$ & $544(59)$ & $1,829(69)$ & $<0.001$ \\
\hline BMI, $\mathrm{kg} / \mathrm{m}^{2}$, mean $\pm \mathrm{SD}$ & $29.2 \pm 9.2$ & $28.6 \pm 11.9$ & $29.4 \pm 8.1$ & 0.027 \\
\hline Alcohol consumer & $105(3.0)$ & $15(1.6)$ & $90(3.4)$ & 0.006 \\
\hline Smoking status & & & & $<0.001$ \\
\hline Current & $876(25)$ & $124(13)$ & $752(29)$ & \\
\hline Recent (>1 month ago) & $75(2.1)$ & $21(2.3)$ & $54(2.1)$ & \\
\hline Past $(>1$ year ago $)$ & $454(13)$ & $94(10)$ & $360(14)$ & \\
\hline Never smoked & $2,154(61)$ & $686(74)$ & $1,468(56)$ & \\
\hline Smoking (current or prior) & $1,405(39)$ & $239(26)$ & $1,166(44)$ & $<0.001$ \\
\hline \multicolumn{5}{|l|}{ Past medical history } \\
\hline Prior MI & $915(26)$ & $191(21)$ & $724(27)$ & $<0.001$ \\
\hline Dyslipidemia & $2,014(57)$ & $462(50)$ & $1,552(59)$ & $<0.001$ \\
\hline Premature CAD & $553(16)$ & $90(10)$ & $463(18)$ & $<0.001$ \\
\hline Hypertension & $2,300(65)$ & $589(64)$ & $1,711(65)$ & 0.483 \\
\hline Diabetes mellitus & $1,896(53)$ & $451(49)$ & $1,445(55)$ & 0.001 \\
\hline Stroke/TIA & $238(6.7)$ & $61(6.6)$ & $177(6.7)$ & 0.896 \\
\hline \multicolumn{5}{|l|}{ Clinical (parameters) at presentation } \\
\hline HR, bpm, mean $\pm S D$ & $85 \pm 21$ & $88 \pm 23$ & $83 \pm 20$ & $<0.001$ \\
\hline $\mathrm{SBP}, \mathrm{mm} \mathrm{Hg}$, mean $\pm \mathrm{SD}$ & $142 \pm 27$ & $143 \pm 27$ & $142 \pm 27$ & 0.317 \\
\hline $\mathrm{DBP}, \mathrm{mm} \mathrm{Hg}$, mean $\pm \mathrm{SD}$ & $81 \pm 16$ & $82 \pm 16$ & $81 \pm 16$ & 0.024 \\
\hline Crea, p50, $\mu \mathrm{mol} / \mathrm{L}$, median (IQR) & $81(66-103)$ & $85(68-108)$ & $81(66-101)$ & 0.002 \\
\hline LVEF, $\%$, mean \pm SD & $49 \pm 13$ & $49 \pm 13$ & $49 \pm 12$ & 0.674 \\
\hline GRACE risk, mean \pm SD & $125 \pm 39$ & $126 \pm 43$ & $125 \pm 38$ & 0.361 \\
\hline CRUSADE*, mean \pm SD & $34 \pm 15$ & $36 \pm 15$ & $34 \pm 15$ & $<0.001$ \\
\hline PCI & $1,026(29)$ & $21(2.3)$ & $1,005(38)$ & $<0.001$ \\
\hline Major bleeding & $40(1.1 \%)$ & $22(2.4)$ & $18(0.7)$ & $<0.001$ \\
\hline Killip class & & & & $<0.001$ \\
\hline I - no heart failure & $2,868(81)$ & $666(72)$ & $2,202(84)$ & \\
\hline II - rales & $440(12)$ & $161(17)$ & $279(11)$ & \\
\hline III - pulmonary edema & $233(6.6)$ & $91(9.8)$ & $142(5.4)$ & \\
\hline IV - cardiogenic shock & $18(0.5)$ & $7(0.8)$ & $11(0.4)$ & \\
\hline Stent type (out of those that had PCI $[n=1,026]$ )* & & & & 0.511 \\
\hline $\mathrm{DE}$ & $833(81)$ & $17(81)$ & $816(81)$ & \\
\hline BM & $154(15)$ & $2(9.5)$ & $152(15)$ & \\
\hline $\mathrm{DE}+\mathrm{BM}$ & $21(2.1)$ & $1(4.8)$ & $20(2.0)$ & \\
\hline Discharged diagnosis & & & & $<0.001$ \\
\hline LBBB MI & $27(0.8)$ & $16(1.7)$ & $11(0.4)$ & \\
\hline NSTEMI & $1,676(47)$ & $430(46)$ & $1,246(47)$ & \\
\hline STEMI & $859(24)$ & $140(15)$ & $719(27)$ & \\
\hline Unstable angina & $997(28)$ & $339(37)$ & $658(25)$ & \\
\hline \multicolumn{5}{|l|}{ Discharged medications $(n=3,559)$} \\
\hline ACEIs & $2,428(68)$ & $638(69)$ & $1,790(68)$ & 0.568 \\
\hline ARBs & $533(15)$ & $110(12)$ & $423(16)$ & 0.002 \\
\hline Beta blockers & $3,040(85)$ & $742(80)$ & $2,298(87)$ & $<0.001$ \\
\hline Statins & $3,462(97)$ & $882(95)$ & $2,580(98)$ & $<0.001$ \\
\hline Other LLDs & $84(2.4)$ & $14(1.5)$ & $70(2.7)$ & 0.048 \\
\hline Oral nitrates & $2,149(60)$ & $620(67)$ & $1,529(58)$ & $<0.001$ \\
\hline CCBs & $536(15)$ & $106(11)$ & $430(16)$ & $<0.001$ \\
\hline
\end{tabular}

BMI was missing in 31 subjects, HR in 2 subjects, SBP in 3 subjects, DBP in 3 subjects, creatinine in 18 subjects, LVEF was missing in 1,225 subjects, GRACE risk score in 20 subjects and 58 subjects in the CRUSADE risk score. Out of those that had PCI, 18 patients had no documented stent types. Percentages might not add up to $100 \%$ due to rounding off. DAPT consisted of aspirin plus clopidogrel; BMI, body mass index; MI, myocardial infarction; CAD, coronary artery disease; TIA, transient ischemic attack; HR, heart rate; bpm, beats per minute; SBP, systolic blood pressure; DBP, diastolic blood pressure; Crea, first serum creatinine; p50 median, IQR, interquartile range; LVEF, left ventricular ejection fraction, ${ }^{*}$ CRUSADE risk score, PCI, percutaneous coronary intervention (includes any prior PCI); LBBB MI, left bundle branch block myocardial infarction; NSTEMI, non-ST myocardial infarction; STEMI, ST myocardial infarction; ACEI, angiotensin-converting enzyme inhibitor; ARB, angiotensin II receptor blocker; LLDs, lipid-lowering drugs; CCBs, calcium channel blockers; DAPT, dual antiplatelet therapy; DE, drug-eluting; $\mathrm{BM}$, bare-metal. 
Table 2. Impact of DAPT (versus aspirin alone) on MACE in patients in the Arabian Gulf with ACS: findings from Gulf COAST registry

\begin{tabular}{|c|c|c|c|c|c|c|c|c|}
\hline \multirow[t]{2}{*}{ Outcome } & \multicolumn{4}{|c|}{ Univariate statistics, $n(\%)$} & \multicolumn{4}{|c|}{ Multivariate logistic regression } \\
\hline & $\begin{array}{l}\text { all } \\
(n=3,559)\end{array}$ & $\begin{array}{l}\text { aspirin } \\
(n=925)\end{array}$ & $\begin{array}{l}\text { DAPT } \\
(n=2,634)\end{array}$ & $p$ value & $\begin{array}{l}\text { adjusted } \\
\text { OR }(95 \% \mathrm{CI})\end{array}$ & $\begin{array}{l}\text { adjusted } \\
p \text { value }\end{array}$ & $\mathrm{HL}$ & ROC \\
\hline Stroke/TIA & $126(3.5)$ & $29(3.1)$ & $97(3.7)$ & 0.438 & $1.68(1.05-2.69)$ & 0.030 & 0.308 & 0.73 \\
\hline Myocardial infarction & $254(7.1)$ & $87(9.4)$ & $167(6.3)$ & 0.002 & $0.66(0.49-0.88)$ & 0.005 & 0.743 & 0.73 \\
\hline All-cause mortality & $273(7.7)$ & $100(10.8)$ & $173(6.6)$ & $<0.001$ & $0.69(0.51-0.94)$ & 0.018 & 0.849 & 0.79 \\
\hline Readmissions & $896(25.2)$ & $272(29.4)$ & $624(23.7)$ & 0.001 & $0.79(0.66-0.95)$ & 0.011 & 0.357 & 0.62 \\
\hline MACE & $1,142(32.1)$ & $359(38.8)$ & $783(29.7)$ & $<0.001$ & $0.73(0.61-0.86)$ & $<0.001$ & 0.315 & 0.66 \\
\hline
\end{tabular}

MACE included stroke/TIA, MI, mortality, and readmissions for cardiac reasons at 12-month follow-up. Events are cumulative. Multivariate analyses were conducted using multiple stepwise logistic regression model utilizing the backward-selection method with a significance $p$ value of 0.2 for the removal from the model. The covariates in the models included GRACE risk score (derived from age, heart rate, systolic blood pressure, serum creatinine, cardiac arrest at admission, ST segment deviation on EKG, abnormal cardiac enzymes, and Killip class) as well as gender, BMI, smoking status, alcohol consumption, diabetes mellitus, peripheral artery disease, dyslipidemia, prior MI, premature coronary artery disease, diabetes mellitus, diastolic blood pressure, major bleeding, percutaneous coronary intervention, discharged cardiac medications, other than aspirin and clopidogrel (beta blocker, statin, ACEI, orARB, CCB), and discharged diagnosis. Over the 1-year follow-up period, there was a total of 3.5\% $(n=125)$ of the patients lost to follow-up. DAPT consisted of aspirin plus clopidogrel adjusted OR, HL Hosmer and Lemeshow $p$ value, ROC area under the receiver-operating curve (also known as c-statistic).

TIA, transient ischemic attack; DAPT, dual antiplatelet therapy; MACE, major adverse cardiac events; MI, myocardial infarction; ACEI, angiotensin-converting enzyme inhibitor; ARB, angiotensin receptor blocker; $\mathrm{CCB}$, calcium channel blocker; aOR, adjusted OR; BMI, body mass index; ST, stent thrombus.

bare-metal (BM) stents, $2.1 \%(n=21)$ had both $\mathrm{DE}$ and $\mathrm{BM}$ stents while 18 patients had no documented stent type. At 1-year follow-up, 3.5\% $(n=125)$ of the patients were lost to follow-up.

As shown in Table 1, those discharged on DAPT were more likely to be males $(p<0.001)$, current smokers $(p<$ $0.001)$, alcohol consumers $(p=0.006)$, or with prior history of MI $(p<0.001)$, dyslipidemia $(p<0.001)$, premature CAD $(p<0.001)$ or diabetes mellitus $(p=0.001)$. Those on DAPT were more likely to have been diagnosed with ST elevation MI (27 vs. $15 \%$; $p<0.001$ ), while those discharged on aspirin alone were more likely to have been diagnosed with unstable angina (37 vs. $25 \%$; $p<0.001$ ). DAPT users were also more likely to have undergone coronary angiography during admission than those discharged on aspirin alone (50 vs. $18 \%$; $p<0.001$ ). However, those discharged on aspirin only presented with higher heart rate ( $88 \mathrm{vs.} 83$ beats/min; $p<0.001$ ), diastolic BP ( 82 vs. $81 \mathrm{~mm} \mathrm{Hg} ; p=0.024)$, serum creatinine ( 85 vs. $81 \mu \mathrm{mol} / \mathrm{L} ; p=0.002$ ), CRUSADE risk score (36 vs. 34; $p<0.001)$, Killip class $(p<0.001)$, and major bleeding during admission (2.4 vs. $0.7 \% ; p<0.001)$. Out of those that were discharged on DAPT, $38 \%(n=988)$ were stented. In those that were on aspirin monotherapy, only $2.2 \%$ $(n=20)$ were stented. At discharge, DAPT patients were also more likely to have been prescribed ARBs (16 vs. $12 \%$; $p=0.002$ ), beta-blockers ( 87 vs. $80 \%$; $p<0.001$ ), statins ( 98 vs. $95 \% ; p<0.001$ ), other lipid-lowering medications ( 2.7 vs. $1.5 \%$; $p=0.048$ ), and CCBs (16 vs. $11 \%$; $p<0.001)$ but less likely to have been prescribed oral nitrates (58 vs. $67 \%$; $p<0.001$ ).

After adjusting for various demographic and clinical confounding factors (Table 2), at 12-months follow-up, patients discharged on DAPT were significantly less likely to experience MACE (adjusted OR [aOR] 0.73; 95\% CI: $0.61-0.86 ; p<0.001)$ compared with patients discharged on aspirin alone. Lower CV risk was also seen across the MACE components: MI (aOR 0.66; 95\% CI: 0.49-0.88; $p=0.005$ ), all-cause mortality (aOR 0.69; 95\% CI: $0.51-$ $0.94 ; p=0.018$ ), and readmissions for cardiac reasons (aOR 0.79; 95\% CI: 0.66-0.95; $p=0.011$ ). However, DAPT was adversely associated with increased risk of stroke/ TIA (aOR 1.68; 95\% CI: $1.05-2.69 ; p=0.030$ ). Out of the 126 stroke/TIAs, only 42 events were strokes, with the rest $(n=84)$ being TIAs. The stroke types were largely missing or unknown (with computer tomography [CTs] not done) with only 1 thrombotic and 1 hemorrhagic stroke documented. 


\section{Discussion}

This large, prospective, multinational, multicenter study from the Arabian Gulf region demonstrates that, at 12-months follow-up, patients with ACS discharged on DAPT compared with those on aspirin alone were significantly less likely to experience MACE outcomes, compared with patients discharged on aspirin alone. Specifically, after multivariate adjustment, ACS patients on DAPT were $34 \%$ significantly less likely to suffer MI, 21\% less likely to be readmitted for any cardiac reason, and $31 \%$ less likely to die. These findings are similar to other studies conducted in other regions of the world using a $\mathrm{P}_{2} \mathrm{Y}_{12}$ receptor inhibitor and aspirin as DAPT $[5,8]$.

The role of DAPT in decreasing the risk of major $\mathrm{CV}$ events, and mortality among ACS and PCI patients was established by several randomized clinical trials such as CURE [5], COMMIT/CCS-2 [6], CLARITY-TIMI-28 [7], and CREDO [14]. DAPT use, with aspirin and clopidogrel, has been shown to reduce 1-year incidence of CV events by approximately $20 \%$ compared with aspirin alone. Since then, more potent and consistent $\mathrm{P}_{2} \mathrm{Y}_{12}$ receptor inhibitors such as prasugrel or ticagrelor have also been used and found to be superior to clopidogrel in TRITON [15] and PLATO [16] trials.

The balance must be struck between reducing future $\mathrm{CV}$ events and increased risk of bleeding due to prolonged use of DAPT $[17,18]$. In both the CURE and PEGASUS-TIMI 54 trials $[5,8]$, DAPT of clopidogrel or ticagrelor with aspirin was associated with 38\% (95\% CI: 1.13-1.67; $p=0.001$ ) and 169\% (95\% CI: $1.96-3.70 ; p<$ 0.001 ) higher risk of major bleeding events compared with aspirin alone, respectively. In the current study, although data on major bleeding 12-months post hospital discharge were not captured, the risk of stroke/TIA was significantly increased by $68 \%$ among patients on DAPT compared with those using aspirin alone. In the recent international POINT study [19], which was halted at $84 \%$ enrolment by the Data Safety Monitoring Board, the combination DAPT regimen was associated with major bleeding including intracranial hemorrhage at 90 days post-initiation of therapy ( 0.9 vs. $0.4 \%$; hazard ratio 2.32 , 95\% CI: $1.10-4.87$; $p=0.02$ ) in patients that had prior minor strokes and TIAs. The authors recommended only short-term use of DAPT followed by monotherapy due to concerns of major bleeding in these patients.

In 2014, the DAPT study demonstrated a decreased risk (30 vs. 12 months of DAPT therapy) of major adverse $\mathrm{CV}$ and cerebrovascular events ( 4.3 vs. $5.9 \%$; $p<0.001$ ), MI (2.1 vs. $4.1 \%$; $p<0.001)$, and ST $(0.4$ vs. $1.4 \%$; $p<$
0.001 ), respectively [20]. However, the benefit of extended DAPT was counterbalanced by an increase in bleeding events ( 2.5 vs. $1.6 \% ; p=0.001$ ), though severe and/or fatal bleeding was uncommon. There was also a borderline rise in all-cause mortality ( 2.0 vs. $1.0 \%$; $p=0.050$ ). However, an analysis comparing this study with 9 other trials enrolling $>20,000$ patients evaluating DAPT duration after DE stent implantation reported that prolonged-DAPT for 12- to 48-month, as compared with short-DAPT for 3- to 12-month, was not associated with significant risk reduction for MI and ST [21].

In arecent meta-analysiscomparingshort ( $\leq 6$-months) vs. prolonged-DAPT ( $\geq 12$-months) duration demonstrated that extension of DAPT beyond 6 months increased the risk of bleeding without reducing the risk of ischemic events [22]. The CURE trial has also shown the greatest absolute risk (average 1\%) and relative risk reductions (average 27\%) were in the first 3 months of DAPT initiation and treatment beyond this period although was useful, but risk reductions were variable ( 0.15 and $12 \%$, respectively) $[18,23]$. Current European and North American clinical guidelines, based on previous studies $[15,16,24]$, recommend DAPT for up to 12 months for ACS patients.

A total of $26 \%$ of the patients were not on the DAPT regimen. However, these results are not unique to the region; similar findings have been reported elsewhere, in Australia [25] and in Denmark [26] where 24 and 32\% of their patients, post-hospital discharge after an ACS event, were on single antiplatelet therapy, respectively. Reasons for not using DAPT included bleeding, neutropenia, thrombocytopenia, vascular complications, and gastrointestinal side effects. It is important to note that this study did not capture the reasons behind the lack of prescribing of the dual DAPT regimen such as side effects, contraindications, allergies, and so on.

This study is not without limitations. First, as this is an observational study, its ability to assess causal relationships is limited. Second, we reported all-cause mortality, whereas CV mortality would have been more appropriate. Third, our study did not capture data on major/minor bleeding events, which made it challenging to compare the safety outcome of DAPT. Nonetheless, strokes/TIAs could be used as surrogates to gauge major bleeding events. Additionally, it would have been more informative if the type of stroke (ischemic or hemorrhagic) was also captured. Fourth, the prevalence of aspirin use only (in the $26 \%$ of the patients) could have been overestimated due to the lack of information on factors such adverse effects, contraindications, and allergies. Fifth, medications were only 
noted at hospital discharge, and they could have been stopped or changed to other medications during the 1 -year follow-up. Sixth, a total of $3.5 \%(n=125)$ of the patients were lost to follow-up at 1 year. However, there were no significant differences in the baseline demographics and clinical characteristics between the group that remained and the one that was lost to follow-up at 1 year.

\section{Conclusions}

To our knowledge, this is the only study in the Arabian Gulf region to evaluate and report on the effect of DAPT on MACE events in ACS patients. At 12-month follow-up after an ACS event, DAPT prescribing was associated with significantly lower MI, readmission for cardiac reasons, and all-cause mortality among Arabian Gulf citizens. However, DAPT was also associated with increased risk of stroke/TIA in ACS patients. The underutilization of the concomitant DAPT regimen in the Arabian Gulf region should alert physicians into bridging the adherence gap in their clinical practice toward evidence-based medicine. These findings should be interpreted in the context of the study's limitations.

\section{Disclosure Statement}

M.Z. and W.A. received speakers' fees from Sanofi, Boehringer Ingelheim, Amgen, and Astra Zeneca. A.A.A.-A. has received honoraria related to consulting and speaker's activities from Boehringer-Ingelheim, Bayer, and Pfizer. I.A.-Z., J.A.-L., A.A.-M., and W.R. have nothing to disclose.

\section{Funding Source}

Gulf COAST is an investigator-initiated study that was supported by Astra Zeneca with an oversight by Kuwait University (Project number XX02/11). Neither Kuwait University nor AstraZeneca had any role in the study design, data collection, data analysis, or writing the manuscript.

\section{Author Contributions}

M.Z. designed and coordinated the study. I.A.-Z. and J.A.-L. wrote the initial draft of the article. M.Z., A.A.A.-A., W.A., W.R., and A.A.-M. were members of the Steering Committee and they conducted the study in their centers. All authors critically reviewed and approved the present article.

\section{References}

1 Ohira T, Iso H. Cardiovascular disease epidemiology in Asia: an overview. Circ J. 2013; 77(7):1646-52.

2 Vedanthan R, Seligman B, Fuster V. Global perspective on acute coronary syndrome: a burden on the young and poor. Circ Res. 2014 Jun;114(12):1959-75.

3 Valgimigli M, Bueno H, Byrne RA, Collet JP, Costa F, Jeppsson A, et al.; ESC Scientific Document Group; ESC Committee for Practice Guidelines (CPG); ESC National Cardiac Societies. 2017 ESC focused update on dual antiplatelet therapy in coronary artery disease developed in collaboration with EACTS: The Task Force for dual antiplatelet therapy in coronary artery disease of the European Society of Cardiology (ESC) and of the European Association for Cardio-Thoracic Surgery (EACTS). Eur Heart J. 2018 Jan;39(3):213-60.

4 Levine GN, Bates ER, Bittl JA, Brindis RG, Fihn SD, Fleisher LA, et al. 2016 ACC/AHA Guideline Focused Update on Duration of Dual Antiplatelet Therapy in Patients With Coronary Artery Disease: A Report of the American College of Cardiology/American Heart Association Task Force on Clinical Practice Guidelines. J Am Coll Cardiol. 2016 Sep;68(10):1082-115.
5 Yusuf S, Zhao F, Mehta SR, Chrolavicius S, Tognoni G, Fox KK; Clopidogrel in Unstable Angina to Prevent Recurrent Events Trial Investigators. Effects of clopidogrel in addition to aspirin in patients with acute coronary syndromes without ST-segment elevation. N Engl J Med. 2001 Aug;345(7):494-502.

6 Chen ZM, Jiang LX, Chen YP, Xie JX, Pan HC, Peto R, et al.; COMMIT (ClOpidogrel and Metoprolol in Myocardial Infarction Trial) collaborative group. Addition of clopidogrel to aspirin in 45,852 patients with acute myocardial infarction: randomised placebo-controlled trial. Lancet. 2005 Nov;366(9497):1607-21.

7 Sabatine MS, Cannon CP, Gibson CM, LópezSendón JL, Montalescot G, Theroux P, et al.; CLARITY-TIMI 28 Investigators. Addition of clopidogrel to aspirin and fibrinolytic therapy for myocardial infarction with ST-segment elevation. N Engl J Med. 2005 Mar; 352(12):1179-89.

8 Bonaca MP, Bhatt DL, Cohen M, Steg PG, Storey RF, Jensen EC, et al.; PEGASUS-TIMI 54 Steering Committee and Investigators. Long-term use of ticagrelor in patients with prior myocardial infarction. N Engl J Med. 2015 May;372(19):1791-800.
9 Zubaid M, Thani KB, Rashed W, Alsheikh-Ali A, Alrawahi N, Ridha M, et al.; Gulf COAST investigators. Design and Rationale of Gulf locals with Acute Coronary Syndrome Events (Gulf Coast) Registry. Open Cardiovasc Med J. 2014 Sep;8(1):88-93.

10 Weintraub WS, Karlsberg RP, Tcheng JE, Boris JR, Buxton AE, Dove JT, et al. ACCF/AHA 2011 key data elements and definitions of a base cardiovascular vocabulary for electronic health records: a report of the American College of Cardiology Foundation/American Heart Association Task Force on Clinical Data Standards. J Am Coll Cardiol. 2011 Jul; 58(2):202-22.

11 Panduranga $\mathrm{P}$, Sulaiman K, Al-Zakwani I, Zubaid M, Rashed W, Al-Mahmeed W, et al. Utilization and determinants of in-hospital cardiac catheterization in patients with acute coronary syndrome from the Middle East. Angiology. 2010 Nov;61(8):744-50.

12 Lemeshow S, Hosmer DW Jr. A review of goodness of fit statistics for use in the development of logistic regression models. Am J Epidemiol. 1982 Jan;115(1):92-106.

13 Hanley JA, McNeil BJ. The meaning and use of the area under a receiver operating characteristic (ROC) curve. Radiology. 1982 Apr; 143(1):29-36. 
14 Steinhubl SR, Berger PB, Mann JT 3rd, Fry ET, DeLago A, Wilmer C, et al.; CREDO Investigators. Clopidogrel for the Reduction of Events During Observation. Early and sustained dual oral antiplatelet therapy following percutaneous coronary intervention: a randomized controlled trial. JAMA. 2002 Nov; 288(19):2411-20.

15 Wiviott SD, Braunwald E, McCabe CH, Montalescot G, Ruzyllo W, Gottlieb S, et al.; TRITON-TIMI 38 Investigators. Prasugrel versus clopidogrel in patients with acute coronary syndromes. N Engl J Med. 2007 Nov;357(20): 2001-15.

16 Wallentin L, Becker RC, Budaj A, Cannon CP, Emanuelsson H, Held C, et al.; PLATO Investigators. Ticagrelor versus clopidogrel in patients with acute coronary syndromes. $\mathrm{N}$ Engl J Med. 2009 Sep;361(11):1045-57.

17 Verdoia M, Kedhi E, Ceccon C, Suryapranata H, De Luca G. Duration of dual antiplatelet therapy and outcome in patients with acute coronary syndrome undergoing percutaneous revascularization: A meta-analysis of 11 randomized trials. Int J Cardiol. 2018 Aug; 264:30-8
18 Wilson SJ, Newby DE, Dawson D, Irving J, Berry C. Duration of dual antiplatelet therapy in acute coronary syndrome. Heart. 2017 Apr; 103(8):573-80.

19 Johnston SC, Easton JD, Farrant M, Barsan W, Conwit RA, Elm JJ, et al.; Clinical Research Collaboration, Neurological Emergencies Treatment Trials Network, and the POINT Investigators. Clopidogrel and Aspirin in Acute Ischemic Stroke and High-Risk TIA. N Engl J Med. 2018 Jul;379(3):215-25.

20 Mauri L, Kereiakes DJ, Yeh RW, Driscoll-Shempp P, Cutlip DE, Steg PG, et al.; DAPT Study Investigators. Twelve or 30 months of dual antiplatelet therapy after drug-eluting stents. N Engl J Med. 2014 Dec;371(23):2155-66.

21 Toyota T, Shiomi H, Morimoto T, Natsuaki M, Kimura T. Short versus prolonged dual antiplatelet therapy (DAPT) duration after coronary stent implantation: A comparison between the DAPT study and 9 other trials evaluating DAPT duration. PLoS One. 2017 Sep;12(9):e0174502.
22 Sharma A, Garg A, Elmariah S, Drachman D, Obiagwu C, Vallakati A, et al. Duration of dual antiplatelet therapy following drug-eluting stent implantation in diabetic and nondiabetic patients: a systematic review and meta-analysis of randomized controlled trials. Prog Cardiovasc Dis. 2018 Jan - Feb;60(4-5): 500-7.

23 Boucher M, Pharand C, Skidmore B. A critical appraisal of the CURE trial: role of clopidogrel in non-ST-segment elevation acute coronary syndromes. Can J Clin Pharmacol. 2004; 11(1):e156-67.

24 Eisenstein EL, Anstrom KJ, Kong DF, Shaw LK, Tuttle RH, Mark DB, et al. Clopidogrel use and long-term clinical outcomes after drug-eluting stent implantation. JAMA. 2007 Jan;297(2):159-68.

25 Anastasius M, Lau JK, Hyun K, D’Souza M, Patel A, Rankin J, et al. The underutilisation of dual antiplatelet therapy in acute coronary syndrome. Int J Cardiol. 2017 Aug;240:30-6.

26 Green $\mathrm{A}$, Pottegård $\mathrm{A}$, Broe $\mathrm{A}$, Diness TG Emneus $M$, Hasvold $P$, et al. Initiation and persistence with dual antiplatelet therapy after acute myocardial infarction: a Danish nationwide population-based cohort study. BMJ Open. 2016 May;6(5):e010880. 